\title{
Radikale onkologische Chirurgie als Therapieprinzip beim Pankreaskarzinom
}

\author{
H. U. Baer, J. M. Läuffer, Ch. Sadowski und M. W. Büchler
}

\section{Schlüsselwörter: Pankreaskarzinom-Chirurgie-Whipple-Operation - Morbidität - Letalität.}

Key-words: Pancreatic cancer-surgery - Whipple-procedure - morbidity - mortality.

Zusammenfassung: Grundlagen: Mit den heutigen tiefen Morbiditäts- und Mortalitätsraten für Pankreasresektionen ist die Indikationsstellung für eine Standard-oder pyloruserhaltende Whipple-Operation weniger restriktiv zu stellen.

Methodik: Pankreasresektionen für Karzinome sollten deshalb in kurativer oder palliativer Absicht, wenn immer möglich, durchgeführt werden. Verlangt werden dafür erfahrene Chirurgen mit über 10 Pankreaseingriffen pro Jahr und einer Mortalität von unter $10 \%$. Als Standard gilt eine 2reihige Pankreatikojejunostomie mit Y-Roux-Rekonstruktion sowie die peri- und postoperative Therapie mit Octreotid während 7 Tagen.

Ergebnisse: Innerhalb von 32 Monaten wurden 113 Patienten mit Pankreaskarzinom behandelt. Die Resektionsrate lag bei $50 \%$, bei der Mehrzahl der Fälle wurde eine pyloruserhaltende Whipple-Operation durchgeführt (30/57). Die postoperative Morbidität lag bei 22,3\%, kein Patient verstarb.

Schlußfolgerungen: Die erweiterte (radikale) Duodenopankreatektomie wird ihren Wert im Vergleich zur Standard- oder pyloruserhaltenden Duodenopankreatektomic in prospektivrandomisierten Studien erst noch belegen müssen. Die Wirksamkeit einer adjuvanten Chemo- und/oder Radiotherapie bedarf weiterer Überprïfung mittels prospektiver Studien.

(Acta Chir. Austriaca 1997;29:267-270)

\section{Extensive Radical Surgery as Therapentic Principal in Patients with Pancreatic Carcinoma}

Summary: Background: The classical and pylorus-preserving Whipple procedure in patients with pancreatic carcinoma can be performed safely with low morbidity and mortality. Therefore, the indication for surgical intervention should be indicated with less restrictions.

Methods: It has been shown that even a palliative resectional procedure has advantages for the patients. Prerequisite to experienced surgeons should be 10 pancreatic resections per year and a mortality $<10 \%$. In order to reduce postoperative typical complications after pancreatic resections in the perioperative period beside antibiotics Octreotid has to be given to inhibit exocrine pancreatic secretion.

Results: Within 32 months 113 patients with pancreatic cancer were treated at out hospital. Resection rate was 50\% (57/113), in the majority of patients a pylorus-preserving whipple procedure was performed (30/57). Postoperative morbidity was $22.3 \%$, no patient died.

Conclusions: The value of extensive and radical pancreatoduodenectomy and the reconstruction according to classical and the pylorus-preserving procedure as well as the role of adjuvant or neoadjuvant chemo- and/or radiotherapy have to be proven in randomized controlled trials. Pancreatic cancer is still a challenging disease for researchers to improve patient's prognosis.

Korrespondenzanschrift: Prof. Dr. M. W. Büchler, Klinik für Viszerale und Transplantationschirurgie, Universität Bern, (Inselspital), Murtenstraße 35, CH-3010 Bern, Schweiz.

\section{Einleitung}

Die Inzidenz der Pankreaserkrankungen hat in den letzten Jahren deutlich zugenommen. Die Gründe dafür mögen im Zusammenhang stehen mit der zunehmenden Überalterung der Bevölkerung sowie mit Fortschritten in der bildgebenden Diagnostik, welche zu einer früheren Entdeckung von Pankreaskarzinomen führen. Das Pankreaskarzinom stellt den Chirurgen deshalb vor vielfältige Probleme bezüglich Indikation und Therapie. Obwohl in den letzten Jahren deutliche Fortschritte in der Pankreaschirurgie erzielt wurden, wird die Indikation zur Operation auch heute noch kontrovers diskutiert (3). Fortschritte in der bildgebenden Diagnostik haben zu einer verbesserten präoperativen Abklärung der Resektabilität von Pankreaskarzinomen geführt. Fortschritte im perioperativen Management - erwähnt sei hier insbesondere die Verabreichung des Somatostatin-Analogs Octreotid (7, 27 $29,30)$ - wie auch in der chirurgischen Technik ermöglichen heute bei großen Pankreasresektionen eine perioperative Mortalität von $5 \%$ und weniger $(8,38)$. Während sich die Resektabilitätsrate in den letzten 10 Jahren von $13 \%$ auf $40 \%$ erhöht hat $(2$, 4,39 ), verbesserte sich das Langzeituberleben aber nur wenig und langsam. Heute bestehen 5-Jahres-Überlebensraten von 10 bis $24 \%(1,4,8,9,31)$. Vielversprechendere Resultate mit 5-Jahres-Überlebensraten von über $30 \%$ wurden für die radikale (erweiterte) Resektion im Frühstadium des Pankreaskarzinoms vor allem aus Japan berichtet $(18,22)$. Dabei wird zusammen mit der Standard-Duodenopankreatektomie eine ausgedehnte retroperitoneale Lymphknoten- und Weichteilresektion durchgeführt. Diese beeindruckenden Resultate berechtigen uns dazu, die Indikation für die Resektion der Pankreaskarzinome neu zu überprüfen.

\section{Operationstechniker}

Für die Pankreaskopfresektion hat Kausch 1912 erstmals eine in 2 Schritten durchgeführte Technik der Resektion publiziert (23). 1935 hat Whipple eine vereinfachte Operation in einem Schritt beschrieben, welche auch heute noch üblich ist (41). Eine neuere Modifikation umfaßt die Erhaltung des Pylorus bei Pankreaskopfresektionen. Als Kontraindikation dieser pyloruserhaltenden Duodenopankreatektomie gilt der Nachweis von positiven gastroepiploischen Lymphknoten, eine Infiltration des proximalen Duodenums mit einem Sicherheitsabstand von weniger als $5 \mathrm{~cm}$ zum Pylorus sowie den Miteinbezug der rechten A. gastrica in den Tumor (34). Falls der bösartige Tumor vom Pankreaskopf bis auf das Korpus oder den Pankreasschwanz übergreift, kann eine totale Pankreatektomie den Tumor entfernen (25). Eine durch Fortner (16) 1984 eingefuhrte und von diversen japanischen Chirurgen modifizierte Technik umfaßt nebst der Standard-Duodenopankreatektomie oder totalen Pankreatektomie eine radikale Lymphadenektomie mit retroperitonealer Weichteilresektion. Oftmals wird auch die V. mesenterica superior, die Portalvene und - seliener - die A. mesenterica superior mirreseziert. Die vaskuläre Rekonstruktion erfolgt entweder mit einer End-zu-End-Anastomose, einem V.-saphena-Interponat oder unter Zuhilfenahme einer Gefäßprothese. Die radikale Lymphadenektomie besteht aus einer weiten retroperitonealen Lymphknotendissektion zwischen dem Ursprung der zöliakalen Achse superior, der iliakalen Bifurkation inferior und den Nieren als laterale Dissektionsbegrenzung.

Somit werden die Lymphknoten von Pylorus, Pankreaskopf und Ductus hepaticus communis sowie die vorderen pankreatikoduodenalen und die oberen mesenterialen Lymphknoten entfernt. Die Entfernung der paraaortalen und zöliakalen Lymphknoten 
sowie die Neurektomie werden en bloc durchgeführt. Die kürzlich publizierten internationalen Resultate der radikalen Pankreatektomie beim Pankreaskopfkarzinom sind in Tabelle 1 zusammengefaßt.

\section{Chirurgische Kriterien der Resektabilität}

Wie bei allen großen chirurgischen Eingriffen, muß vorgängig eine Operabilitätsabklärung erfolgen. In erster Linie müssen der Allgemeinzustand und die allgemeinen Risikofaktoren des Patienten berücksichtigt werden. Ein hohes Alter ist für sich allein genommen nicht als Kontraindikation anzusehen. Es muß aber zusammen mit anderen Risikofaktoren berücksichtigt werden. Der Wert des histologischen Nachweises von Karzinomgewebe mittels Feinnadelpunktion ist für die Indikationsstellung zur Operation eher gering, da neuere Untersuchungen eine geringere Sensitivität und Spezifität ergeben haben als früher angenommen wurde $(15,36)$. Weiterhin muß der Chirurg die technischen Möglichkeiten einer Resektion abschätzen. Eine Resektion kann dabei kurativ unternommen werden mit der Absicht, alles sichtbare Tumorgewebe zu entfernen, oder in palliativer Absicht, wenn eine komplette Resektion des Tumors nicht mehr möglich ist. Die chirurgischen Möglichkeiten hängen weiter ab von der Lokalisation des Tumors im Pankreas. In 60 bis $70 \%$ werden die Karzinome im Pankreaskopf gefunden (20). Sie komprimieren die intrapankreatischen Gallenwege und den Ductus pancreaticus früh und infiltrieren Nervengewebe, Gefäße und regionale Lymphknoten.

Später bilden sie Leber- und andere extrapankreatische Metastasen. In 20 bis $30 \%$ der Pankreastumoren wird der Pankreaskorpus und in 5 bis $10 \%$ der Schwanz des Pankreas erfaßt. Zur Zeit der Diagnosestellung sind diese Tumoren normalerweise groß und haben die Pankreasgrenze bereits überschritten. Sie infiltrieren meist sehr früh ins Retroperitoneum, in angrenzende Organe sowie in Nerven, Gefäße und Lymphknoten. Häufig werden auch Leber- und Peritonealmetastasen gefunden. Die Resektabilität für Pankreaskorpus- und -schwanztumoren ist deshalb beschränkt und das Langzeitüberleben der Ausnahmefall. Johnson et al. fanden in einer Serie von 105 Patienten mit Pankreaskarzinomen im Korpus- und Schwanzbereich eine Resektabilitätsrate von nur $12 \%$ mit nur einigen wenigen Langzeitiberlebenden (22).

Die präoperative Untersuchung der Resektabilität wird heute mit bildgebenden Verfahren durchgeführt. Diese umfassen Ultraschall, Computertomographie (CT) und Magnetic Resonance Imaging (MRI). Sie stellen die exakte Lage und Ausdehnung des Tumors sowie Infiltration in die umliegenden Organe dar. Die Cholangiopankreatographie (ERCP) zeigt den Befall von intraund extrapankreatischen Gallenwegen sowie des Ductus pancreaticus. Eine Angiographie ist nicht indiziert, da die Sensibilität von CT und MRI für den Nachweis eines Gefäßbefalls ausreichend hoch ist. Die Kombination dieser 2 bildgebenden Verfahren hilft in vielen Fällen bereits präoperativ, eine Irresektabilität festzustellen. In den meisten Fällen jedoch bleibt die definitive Entscheidung, ob ein Tumor reseziert werden kann oder nicht, der Laparotomie (oder der Laparoskopie) vorbehalten.

Tab. 1. Radikale (erweiterte) Pankreatektomie beim Pankreaskopfkarzinom.

\begin{tabular}{|c|c|c|c|c|c|}
\hline Antor & Jahr & $\underline{m}$ & Morbidität & Mortallitäí & Uberleben \\
\hline Hailabe et al. & 1985 & 21 & unbekannt & $14 \%$ & $0 \% 5$ jahre \\
\hline Tsuchiya et al. & 1986 & 106 & unbekannt & $4,0 \%$ & $30,3 \% 5$ Jahre \\
\hline Ishikawa et al. & 1988 & 59 & unbekannt & $10.0 \%$ & $9 \% 5$ Jahre \\
\hline Tashiro et al. & 1991 & 17 & $18 \%$ & $5,0 \%$ & $6 \% 5$ Jahre \\
\hline Nagakawa et al. & 1991 & 66 & unbekannt & $4,5 \%$ & $3 \% 5$ Jahre \\
\hline Nagakawa et al. & $199 !$ & 61 & $37,2 \%$ & $6,1 \%$ & $26.4 \% 5$ Jahre \\
\hline Ishigawa et al. & 1992 & 35 & unbekannt & $6,0 \%$ & $23 \% 3$ Jahre \\
\hline Satake et al. & 1992 & 57 & unbekannt & $7,0 \%$ & $28.3 \% 5$ Jahre \\
\hline Gotoh et al. & 1992 & 17 & $41,2 \%$ & $0 \%$ & unbekarnt \\
\hline Hanyu et al. & 1993 & 167 & unbekannt & $5.3 \%$ & $8 \% 5$ Jahre \\
\hline
\end{tabular}
2 Faktoren abhängig gemacht werden: vom Patienten und vom Chirurgen. Sowohl Tumorstadium und -ausdehnung als auch die technische Fähigkeit. Erfahrung und Aggressivität des Chirurgen beeinflussen das operative Vorgehen. Objektive Faktoren der Irresektabilität sind komplexer Gefäßbefall, Infiltration ins Retroperitoneum und Fernmetastasen (Tab. 2). Der Gefäßbefall kann die Portalvene, die V. mesenterica superior, die zöliakale Achse sowie die Leberarterie und Milzgefäße umfassen. Pankreastumoren mit retropankreatischer Infiltration der Wand der V. mesenterica superior oder der Portalvene können immer noch resektabel sein. Die Gefäßvorderwand kann reseziert und direkt mit fortlaufender Naht verschlossen werden, oder das Gefäß wird komplett reseziert und eine direkte porto-portale Anastomose wird durchgeführt. In Fällen von retroperitonealer Infiltration ist eine radikale Lymphadenektomie mit ausgedehnter Weichteilresektion im Sinne der radikalen (erweiterten).

Im Einzelfall muß der verantwortliche Chirurg in seiner Beurteilung nicht nur Ausdehnung und Größe der möglichen Resektion, sondern auch seine eigenen chirurgisch-technischen Erfahrungen miteinbeziehen. Grundvoraussetzung für eine erfolgreiche Pankreaschirurgie sind gut ausgebildete und spezialisierte Chirurgenteams, welche im Minimum 10 Pankreasresektionen pro Jahr durchführen und eine perioperative Mortalität unter $10 \%$ nachweisen können (9).

Dabei werden die besten Resultate von Kliniken mit einer hohen Anzahl Resektionen pro Jahr erreicht (21).

\section{Ergebnisse}

Wir haben von November 1993 bis Juni 1996 unsere Patienten nach einem standardisierten prospektiven Protokoll behandelt. Präoperativ wurden die Patienten mit einer CT zur Beurteilung des Parenchyms und einer ERCP zur Beurteilung des Ductus pancreaticus abgeklärt. Wir haben eine möglichst blutsparende Resektions- und Präparationstechnik eingesetzt und ausschließlich Pankreatikojejunostomien mit einer Y-Roux-Rekonstruktion durchgeführt. Die Pankreasanastomose wurde dabei in 2 schichtiger Nahttechnik nach Möglichkeit mit einer End-zu-Seit-Anastomose durchgeführt. Dies insbesondere bei großer Diskrepanz zwischen Pankreas- und Jejunumdurchmesser oder bei weichem Pankreasparenchym. Alle Patienten erhielten Octreotid für 7 Tage in der Dosierung von $3 \times 100 \mathrm{~g}$ subkutan. Wir haben 113 Patienten, 57 Männer und 56 Frauen. bis Juni 1996 in der Studie prospektiv erfaßt. Das Durchschnittsalter war 66 (Range 33 bis 84) Jahre. Die Indikation zur Pankreasresektion wurde bei allen Patienten wegen eines Pankreaskarzinoms gestellt. 102 waren im Kopf. 6 im Korpus und 5 im Pankreasschwanz lokalisiert. Die durchgeführten Operationen sowie die Morbidität sind in Tabelle 3 zusammengefaßt. Todesfülle sind keine aufgetreten.

\section{Diskussion}

Die radikale (erweiterte) Pankreatektomie ist in den letzten Jahren als Therapie für das Pankreaskarzinom diskutiert und von einigen Autoren angewendet worden (Tab. 1) Früher herrschte die Meinung vor, dab infolge der hohen operativen Mortalität und des schlechten Langzeitiberlebens keine Indikation für eine Resektion von Pankreaskarzinomen besteht (10, 12). Ersî als Resultate der letzten Dekade wie auch unsere eigenen zeigten, daß diese Aussage heute nicht mehr zutrifft, wurden die Indikationen zur Pankreasresektion und zu erweiterten Resektionen großzügiger gestellt. Mit zunehmender Erfahrung und Verbesserung der technischen Fähigkeiten der Chirurgen kann heute auch die ausgedehnte Pankreaschirurgie sicher durchgeführt werden. In Zentren mit großer Erfahrung wird eine Mortalität von unter $5 \%$ erreicht $(8,11,20,38)$. In unserer ei- 
Tab. 2. Absolute und relative Kontraindikationen (1).

\begin{tabular}{|c|}
\hline Klare Kontraindikationen \\
\hline inoperabler Patient (kardiopulmonal) \\
\hline Lebermetastasen \\
\hline Peritonealmetastasen \\
\hline komplexe Gefäßinvasion (Venen und Arterien) \\
\hline Relative Kontraindikationen \\
\hline Infiltration des Retroperitoneums \\
\hline Infiltration der V. mesenterica superior \\
\hline Infiltration der Portalvene \\
\hline
\end{tabular}

genen Serie von 113 Pankreasresektionen haben wir keine Mortalität und eine Morbidität von $18 \%$ bei nur einer vorïbergehenden Pankreasfistel $(0,7 \%)$ beobachtet. Unsere Technik der Pankreasanastomose End-zu-End oder End-zu-Seit mit einer YRoux-Anastomose ist sicher und erreicht gute Resultate. Neben der chirurgischen Technik halten wir auch die peri- und postoperative Behandlung mit Octreotid, zur Hemmung der exokrinen Sekretion für die Verhinderung von Komplikationen für wichtig. Diese Therapie reduziert die Morbidität, die in unseren Serien vergleichbar ist mit andern Serien, bei welchen routinemäßig Octreotid eingesetzt wurde $(27,29,30)$. Castillo et al. (14) erreichten zum Beispiel bei 231 Pankreasresektionen, darunter 142 Duodenopankreatektomien und 71 distale Pankreatektomien, eine Mortalität von $0,4 \%$.

Die Morbidität bestand vor allem in einer verzögerten Magenentleerung $(6,9 \%)$ und Bildung von Pankreasfisteln $(7,5 \%)$. Diese Serie, wie auch unsere eigenen Resultate, zeigen, daß heute eine Pankreasanastomose mit großer Sicherheit durchgeführt werden kann. Deshalb kann heute in der Pankreaschirurgie auch erweitert reseziert werden.

Die totale Pankreatektomie, durchgeführt, um eine Pankreasanastomose zum Dünndarm zu verhindern, ist aufgrund der beträchtlich höheren Morbidität und Mortalität nur noch in Spezialfällen, vor allem bei diffusem Karzinombefall des ganzen Pankreas indiziert $(2,6,40)$. Die meisten Chirurgen stimmen heute darin überein, daß Fernmetastasen und komplexe Gefäßinfiltrationen eine klare Kontraindikation zur Pankreasresektion darstellen. Für uns besteht aber auch dann noch eine Operationsindikation, wenn die präoperative bildgebende Abklärung scheinbar eine Infiltration des Retroperitoneums zeigt. Erst die intraoperative Exploration kann diese Infiltration bestätigen oder ausschließen und aufzeigen, ob eine palliative Resektion mit allenfalls postoperativer regionaler Chemotherapie angezeigt ist.

Für die Whipple-Operation werden heute 5-Jahres-Überlebenszeiten von 10 bis $36 \%$ erreicht $(4,12,31,38)$. Die mediane Überlebenszeit liegt zwischen 11 und 18 Monaten $(4,31,32)$. Wenn die Resektion für Karzinome mit weniger als $2 \mathrm{~cm}$ Durchmesser erfolgt, so zeigt eine japanische Multizenterstudie eine 5Jahres-Überlebenszeit von $30 \%$ (39). Bei Tumoren im Stadium I wurden sogar Überlebenszeiten von $39 \%$ nach kurativer Resektion erreicht (8).

Obwohl nur eine Minorität der Patienten in diesem frühen Stadium diagnostiziert wird, zeigen diese Resultate, daß die Chirurgie heute gute Resultate erreichen kann. Spätkomplikationen werden nur selten beobachtet und häung erst in der meist kurzen präterminalen Phase gefunden. Es scheint deshalb, daß die Uberlebenszeit und die Lebensqualität nach der Pankreasresektion verbessert sind. Dies trifft insbesondere für die pyloruserhaltende Pankreaskopfresektion zu. Die Antrum-Pylorus-Einheit bleibt dabei erhalten und ermöglicht eine optimale postoperative Ernährung (34). Die Patienten können nach dem Eingriff wieder ein normales Leben führen, erreichen normalerweise sehr schnell ihr Normalgewicht wieder und haben eine ausgezeichnete Lebensqualität $(5,24)$. Mit einer tiefen Mortalität, wie sie heute für die Whipple-Operation in erfahrenen Händen erreicht werden kann. scheint die Resektion auch für palliative Fälle gerechtfertigt. Da die extrapankreatische Verbreitung der Pankreaskarzinome über
Tab. 3. Pankreaskarinom Viszeralchirurgie Inselspital.

\begin{tabular}{|l|c|}
\hline & Anzahl \\
\hline Total & 113 \\
\hline Geschlecht & \\
\hline Männer & 57 \\
\hline Frauen & 66 \\
\hline Alter (Jahre) & \\
\hline Durchschnitt & 66 \\
\hline Range & $(33-84)$ \\
\hline Diagnose & \\
\hline Pankreaskopfkarzinom & 102 \\
\hline Pankreaskorpus & 6 \\
\hline Pankreasschwanz & 5 \\
\hline Operationen für Pankreaskarzinome & \\
\hline Resektionen & 57 \\
\hline nach Whipple & 17 \\
\hline pyloruserhaltende Whipple-Resektion & 30 \\
\hline Linksresektion & 5 \\
\hline totale Pankreatektomie & 5 \\
\hline Bypass & 36 \\
\hline explorative Laparotomien & 20 \\
\hline Morbidität & $22.3 \%$ \\
\hline Letalität & 0 \\
\hline
\end{tabular}

Nerven, Blut- und lymphatische Gefäße sehr früh stattfindet, werden die meisten Patienten erst in vorgeschrittenen Stadien diagnostiziert und reseziert $(13,32)$. Autopsiestudien haben ergeben, daß auch kleine Pankreastumoren in $40 \%$ der Patienten bereits positive paraaortale Lymphknoten haben (28). Weitere Studien zeigen, daß über 50\% der resezierten Patienten ein Lokalrezidiv entwickeln $(35,37)$. Diese Beobachtungen scheinen zu belegen, daß eine Standard-Whipple-Operation allein ein Tumorrezidiv nicht verhindern kann; ob erweiterte Resektionen dies können, ist nicht bewiesen. Die heutigen Resultate nach radikaler (erweiterter) Resektion sind noch widersprüchlich.

Ishikawa et al. (19) zeigten in einer retrospektiven Studie eine 3-Jahres-Überlebensrate von $13 \%$ bei 32 Patienten nach StandardDuodenopankreatektomie vs. $38 \%$ bei 21 Patienten nach erweiterter Duodenopankreatektomie. Manabe et al. (26) führten bei 42 Patienten eine Standard-Whipple-Operation durch mit einer operativen Mortalität von $9,5 \%$ bei keinem Überlebenden nach 3 Jahren. $32 \mathrm{~Pa}$ tienten erhielten eine erweiterte Duodenopankreatektomie. Die operative Mortalität war mit 6,2\% deutlich niedriger und das 5-JahresÜberleben mit 33,5\% klar erhöht. Im Gegensatz dazu sei die Studie von Satake et al. (33) erwähnt. Sowohl bei der erweiterten als auch bei der Standardresektion wurden bei 185 Patienten mit einer Tumorgröße von weniger als $2 \mathrm{~cm}$ die gleiche 5-Jahres-Überlebensraten von $27 \%$ gefunden. Ein ähnliches Resultat wurde von Geer et al. (17) berichtet mit einer medianen Überlebenszeit von $18 \mathrm{Mo}$ naten nach Standardresektion und 22 Monaten nach erweiterter Resektion. Die Morbidität und Mortalität nach erweiterter Resektion scheinen identisch zu sein wie bei der Standardresektion. Die Morbidität liegt zwischen 20 und $40 \%$, die Mortalität zwischen 4 und $14 \%$ (Tab. 1). Sepsis und Hämorrhagie sind die Haupttodesursachen. Verschiedene Autoren berichten auch über postoperatives Leberversagen aufgrund einer Gefäßthrombose nach vaskulärer $\mathrm{Re}$ konstruktion. Erst prospektive und randomisierte Studien, welche die erweiterte Resektion mit der Standard-Whipple-Operation vergleichen, könnten den Wert der erweiterten Doudenopankreatektomie definitiv belegen. Die Whipple-Operation bleibt deshalb heute noch die Standardresektion, gegen welche alle andern Techniken verglichen werden müssen.

Ebenfalls sind weitere prospektive Studien nötig, um den Wert einer möglichen adjuvanten Chemotherapie, eventuell in Kombi- 
nation mit einer - auch intraoperativen - Strahlentherapie, zu belegen. Die heutigen tiefen Mortalitätsraten nach Pankreasresektionen rechtfertigen aber in jedem Fall eine neue, etwas aggressivere Haltung gegenüber dem Pankreaskarzinom. Unserer Meinung nach ist auch die Indikation für eine palliative Pankreaskopfresektion heute zumindest gut zu überlegen, da eine Resektion die beste Palliation der quälenden Symptome des Ikterus und der Pankreasgangstauung sind.

\section{Literatur}

(1) Aigner KR, Müller H, Bassermann R: Intra-arterial chemotherapy with MMC. CDDP and 5-FU for nonresectable pancreatic cancer - a phase II Study. Reg Cancer Treat 1990;3:1-6

(2) Andren-Sandberg A, Ihse I: Factors influencing survival after total pancreatectomy in patients with pancreatic cancer. Ann Surg 1993;198:605.

(3) Baer HU, Wagner M, Büchler MW: Pancreatic cancer: indications for surgery. (3) Baer HU, Wagner M, B
Dig Surg 1994;11:372-377.

(4) Beger HG, Büchler MW, Friess H: Chirurgische Ergebnisse und Indikation zu adjuvanten Maßnahmen beim Pankreascarcinom. Chirurg 1994;65:246.

(5) Braasch JW, Gagner M: Pylorus-preserving pancreato-dudenectomy Technical aspects. Langenbecks Arch Chir 1991;376:50.

(6) Brooks JR: the case for total pancreatectomy: in the Delaney JP, Varco RL (eds): Controversy in Surgery. Part II. Philadelphia, Saunders, 1983, pp 327.

(7) Büchler M, Friess H, Klempa I, Hermanek P, et al: The role of octreotide in the prevention of postoperative complications following pancreatic resection. Am J Surg

(8) Büchler MW, Ebert M, Beger HG: Grenzen chirurgischen Handelns beim Pankreaskarzinom. Langenbecks Arch Chir 1993;75 (suppl)

(9) Cameron JL, Crist DW. Sitzmann JV: Factors influencing survival after total pancreatectomy in patients with pancreatic cancer. Ann Surg 1983;198:605.

(10) Carter DC: Surgery for pancreatic cancer. Br Med J 1980;1:774.

(11) Conlon KC. Klimstra DS, Brennan MF: Long-term survival after curative resection for pancreatic ductal adenocarcinoma: clinicopathologic analysis of 5-year survivors. Ann Surg 1996:223:273-279.

(12) Crist DW, Sitzmann JV, Cameron JL: Improved hospital morbidity, mortality and survival after the Whipple procedure. Ann Surg 1987;206:358.

(13) Cubilla AC, Fortner J, Fitzgerald PJ: Lymph node involvement in carcinoma of the head of the pancreas area. Cancer 1978:41:880.

(14) Fernàndez-del Castillo C. Rattner DW, Warshaw AL: Standards for pancreatic resection in the 1990s. Arch Surg 1995;130:295.

(15) Forsmark CE, Albert CA, Lambiase L, Vogel SB: Diagnostic tests for pancreatic cancer. Gastrointest Endosc 1993;9:A314.

(16) Fortner JG: Regional resection of cancer of the pancreas: a new surgical (16) Fortner JG: Regional resection

approach. Surgery 1973;73:307-320.
(17) Geer RJ, Brennan MF: Prognostic indicators for survival after resection of pancreatic adenocarcinoma. Am J Surg 1993;165:68-73.

(18) Hiraoka T: Extended radical resection of cancer of the pancreas with intraoperative radiotherapy. Baill Clin Gastroenterol 1990;4:889.

(19) Ishikawa O, Ohhigashi $H$, Sasaki Y: Practical usefulness of lymphatic and connective tissue clearance for carcinoma of the pancreas head. Ann Surg 1988:208:215-220.

(20) Janes RH, Niederhuber JE, Chmiel JS, Winchester DP. Ocwieja KC. Karnell LH. Clive RE, Menck HR: National patterns of care for pancreatic cancer: results of a survey by the commission on cancer. Ann Surg 1996;223:261-272.
(21) Janes RH, Niederhuber JE, Chmiel JS, Winchester DP, Ocwieja KC, Karnell LH. Clive RE, Menck HR: National patterns of care for pancreatic cancer: results of a survey by the commission on cancer. Ann Surg 1996;223:261-272.

(22) Johnson CD. Schwall G, Flechtenmacher J, Trede M: Resection for adenocarcinoma of the body and tail of the pancreas. Br J Surg 1993:80:1177-1179.

(23) Kausch W: Das Carcinom der Papilla duodeni und seine radikale Entfernung. Beitr Klin Chir 1912:78:439.

(24) Klinkenbijl JHG, Van der Schelling GP, v Hop WCJ, Van Pel R, Bruining HA Jeckel $\mathrm{J}$ : The advantages of pylorus-preserving pancreatoduodenectomy in malignant disease of the pancreas and the periampullary region. Ann Surg 1992;216:142. (25) Launois B, Franci J, Bardaxoglu E, et al: Total pancreatectomy for ductal adenocarcinoma of the pancreas with special reference to resection of the portal vein and multicentric cancer. World J Surg 1993;17:122

(26) Manabe T, Suzuki T. Tobe T: Evaluation of en bloc radical pancreatectomy for carcinoma of the head of the pancreas involving the adjacent vessels. Dig Surg $1985 ; 2: 27-30$

(27) Montorsi M, Zago M. Mosca F, Capussotti L, Zotti E, Ribotta G, Fegiz G, et al Efficacy of octreotide in the prevention of pancreatic fistula after elective pancreatic resections: a prospective, controlled, randomized clinical trial. Surgery 1995;117:2631.

(28) Nagai H, Kuroda A. Morioka Y: Lymphatic and local spreading of T1 and T2 pancreatic cancer. Ann Surg 1986;204:65.

(29) Paran H, Neufeld D. Mayo A, Shwartz I, Singer P, Kaplan O, Skornik Y, Klausner J, Freund U: Preliminary report of a prospective randomized study of octreotide in the treatment of severe acute pancreatitis. J Am Coll Surg 1995;181:121124.

(30) Pederzoli P, Bassi C, Falconi M, Camboni MG: Efficacy of octreotide in the prevention of complications of elective pancreatic surgery. Br J Surg 1994;81:265269.

(31) Reber HA: The Whipple pancreaticoduodenectomy for pancreatic cancer. In Beger HG, Büchler MW, Malfertheiner P (eds): Standards in Pancreatic Surgery. Berlin, Springer, 1993, pp 637.

(32) Russel RCG: Surgical resection for cancer of the pancreas. Baill Clin Gastroenterol 1990;4:889.

(33) Satake K, Nishiwaki H, Yokomatsu H, Kawazoe Y, Kim K, Haku A, Umeyama K, Miyazuki I: Surgical curability and prognosis for standard versus extende resection for Tl carcinoma of the pancreas. Surg Gynecol Obstet 1992;175:259.

(34) Seiler CA, Wagner M, Büchler MW: The role of pylorus-preserving duodenopancreatectomy in pancreatic cancer. Dig Surg 1994:11:390-396.

(35) Sindelar WF: Clinical experience with regional pancreatectomy for adenocarcinoma of the pancreas. Arch Surg 1989;124:127.

(36) Swobodnik W, Janowith P. Beck J: Abhängigkeit der Aussagekraft ultraschallgesteverter diagnostischer Feinnadelpunktionen von Größe, Lokalisation und Echocharakteristika des Punktionsziels. Ultraschali Klin Prax 1991;5:241.

(37) Tepper T, Nardi G. Suit H: Carcinoma of the pancreas: Review of MGH experience from 1963 to 1973. Analysis of surgical failure and implications for radiation therapy. Cancer 1976;37:1519.

(38) Trede M, Schwall G, SaEger HD: Survival after pancreatoduodenectomy. Ann Surg 1990;211:447.

(39) Tsuchiya R, Tsunoda T, Ishida T, Saitoh Y: Resection for cancer of the pancreas - The Japanese experience. Baill Clin Gastroenterol 1990;4:931

(40) van Heerden JA: Pancreatic resection for carcinoma of the pancreas: Whipple versus total pancreatectomy - An institutional perspective. World J Surg 1984;8:880. (41) Whipple AO, Parsons WB, Mullins CR: Treatment of carcinoma of the ampulla of Vater. Ann Surg 1935;102:763.

\section{Kongreßankündigungen}

\section{Internationales Symposium für Kinderchirurgie}

Termin und Ort: 19. bis 21. Jänner 1998 - Obergurgl. Organisation: Univ.-Prof. Dr. Ernst Horcher, Universitätsklinik für Chirurgie, Klinische Abteilung für Kinderchirurgie, Währinger Gürtel 18-20, A-1090 Wien, Tel. $01 / 40400$ DW 6836, Fax DW 6838.

\section{Fotoworkshop für intra- und perioperative Fotografie für Ärzie und medizinisches Personal}

Terrain und Ort: 10. Februar 1998 - Graz

Organisation: Dr. $H$. Clement, Universitätsklinik für Unfalichirurgie, Auenbruggerplatz 7a, A-8036 Graz, Tel. +43/316/ 385 DW 3547 (Frau Bloder oder Frau Greiner), Fax DW 3582.

\section{Grazer Workshop: Unfallchirurgische Notfalleingriffe mit praktischen Übungen}

Termin und Ort: 14. Februar 1998 - Graz.

Organisation: Doz. Dr. M. Fellinger und Dr. W. Grechenig, Universitätsklinik für Unfallchirurgie, Auenbruggerplatz 7a, A-8036 Graz, Tel. +43/316/385 DW 3547 (Frau Bloder oder Frau Greiner), Fax DW 3582.

\section{Colorectal Disease 1998}

Termin und Ort: 19. bis 21. Februar 1998 - Fort Lauderdale. Florida, USA.

Kongreßsekretariat: Kathy Warren, Cleveland Clinic Florida, Department of Education, 2950 West Cypress Creek Road, Fort Lauderdale, FL 33309-1743, USA, Tel. 001954 / 978 DW 5529, Fax DW5757 oder 5539.

Offene und laparoskopische chirurgische Techniken 15. Praktischer Kurs für Gastroenterologische Chirurgie Termin and Ort: 28. Februar bis 6. März 1998 - Davos, Schweiz.

Information: AGC-Kurs, V. Bertschi, clo ISS/SIC. Netzibodenstraße 34, Postfach 1527, CH-4133 Pratteln, Tel. +41/61/ 811 DW 4772, Fax DW 4775.

\section{Viszeralchirurgische Woche}

Termin and Ort: 14. bis 21. März 1998 - Wagrain. Haupthema: Komplikationsvermeidung und -behandlung in der konventionellen und videoendoskopischen Viszeralchirurgie.

Organisation und Information: Prof. Dr. H. W. Waclawiczek, A.ö. Landeskrankenanstalten Salzburg, I. Chirurgische Abteilung, Müllner Hauptstraße 48, A-5020 Salzburg. Tel. $+43 / 662$ / 4482 DW 3103, Fax DW 3104. 